\title{
ALMOST SPLIT SEQUENCES FOR RATIONAL DOUBLE POINTS
}

\author{
MAURICE AUSLANDER ${ }^{1}$ AND IDUN REITEN
}

\begin{abstract}
Let $R$ be a complete local two-dimensional integrally closed noetherian nonregular Gorenstein domain with maximal ideal $m$ over an algebraically closed field $k$, such that $R / m \simeq k$. Then the category of finitely generated reflexive modules is known to have almost split sequences, and our main result is that the $A R$-quiver is an extended Dynkin quiver $\tilde{A}_{n}, \tilde{D}_{n}, \tilde{E}_{6}, \tilde{E}_{7}$ or $\tilde{E}_{8}$.
\end{abstract}

Let $R$ be a complete local two-dimensional integrally closed noetherian nonregular Gorenstein domain with maximal ideal $m$ over an algebraically closed field $k$, such that $R / m \simeq k$. Denote by $\mathbf{L}$ the category of finitely generated reflexive modules, or equivalently, the (maximal) Cohen-Macaulay modules over $R$. Then $\mathbf{L}$ is known to have almost split sequences $[6,3,4]$, and we prove that if $R$ is of finite Cohen-Macaulay type, then the $A R$-quiver for $\mathbf{L}$ is an extended Dynkin quiver $\tilde{A}_{n}$ $\tilde{D}_{n}, \tilde{E}_{6}, \tilde{E}_{7}$, or $\tilde{E}_{8}$, in a sense explained in $\S 1$. If the vertex corresponding to $R$ is removed, we get the corresponding Dynkin quiver.

The rational double points (over an algebraically closed field $k$ ) satisfy the above hypothesis, including finite Cohen-Macaulay type [2]. If the characteristic of $k$ is 0 , these are known to be the fixed point rings $k[[X, Y]]^{G}$, where $G \subset \operatorname{SL}(2, k)$ is a finite nontrivial group and the action is the natural one. In this case a proof for the above result was given by showing that the $A R$-quiver is isomorphic to the McKay quiver for $G \subset \operatorname{SL}(2, k)[4]$, which was already known to be an extended Dynkin quiver [17]. Here we give a direct proof valid for any characteristic. We use additive functions, as in [15] for McKay quivers, the fundamental "almost split" sequence from $[3,4]$, preprojective partitions from [10], and the computation of Grothendieck groups using almost split sequences (see [8]).

The extension to arbitrary characteristic is of interest in connection with resolution of singularities, where for a rational double point the resolution graph is known to be Dynkin. In particular this work is used in [13] to prove that there is an isomorphism between the graph given by the $A R$-quiver after removing $R$, and the resolution graph, also in characteristic 2,3 , and 5 .

We also give a generalization to the noncommutative two-dimensional case, for lattices over a $T$-order $\Lambda$ when $T$ is a complete regular local ring with maximal ideal $m$ and $T / m$ is algebraically closed. We here get as possibilities some additional $A R$-quivers with loops. In the last section we compute the $A R$-quivers for some noncommutative rings shown to be of finite lattice type in [1]. These computations show that the cases with loops actually do occur.

Received by the editors January 31, 1986.

1980 Mathematics Subject Classification (1985 Revision). Primary 13H10; Secondary 16A18.

${ }^{1}$ Written with partial support from NSF. 
We would like to thank Øyvind Solberg for his help with calculations and careful reading of the manuscript.

1. The main theorem. In this section we shall deal with a complete local nonregular integrally closed noetherian two-dimensional Gorenstein domain $R$, with maximal ideal $m$, and study the category $\mathbf{L}$ of reflexive $R$-modules, which is known to have almost split sequences $[3,4]$. For such a category denote for $A$ and $B$ indecomposable in $\mathbf{L}$ by $r_{A B}$ the multiplicity of $A$ in the minimal right almost split map for $B$, and by $s_{A B}$ the multiplicity of $B$ in the minimal left almost split map for $A$. Then we recall that the $A R$-quiver $\Gamma$ for $\mathbf{L}$ has as vertices the isomorphism classes of indecomposable reflexives, and there are $r_{A B}=s_{A B}$ arrows from $A$ to $B$. This holds when all $\operatorname{End}(A) / \operatorname{rad} \operatorname{End}(A)$ are isomorphic to the same algebraically closed field $k$ (see [11]), which is the case under the assumptions of the next theorem. We denote by $\Gamma_{s}$ the quiver obtained by removing the vertex corresponding to the projective module $R$. If in a quiver $\Gamma$ the arrows between different vertices occur in pairs $\rightleftarrows$, we denote by $|\Gamma|$ the graph obtained by replacing $\cdot \rightleftarrows \cdot$ by $\cdot-\cdot$ and $\Im$ by $C$.

We have the following result on the $A R$-quiver.

THEOREM 1. Let $R$ be a complete nonregular integrally closed local two-dimensional noetherian Gorenstein domain with $R / m=k$ algebraically closed, assume that $R$ has only a finite number of isomorphism classes of indecomposable reflexive modules, and let $\Gamma$ be the corresponding AR-quiver.

The arrows in $\Gamma$ occur in pairs $\cdot \rightleftarrows \cdot$, and $|\Gamma|$ is an extended Dynkin diagram $\tilde{A}_{n}$, $\tilde{D}_{n}, \tilde{E}_{6}, \tilde{E}_{7}$, or $\tilde{E}_{8} .\left|\Gamma_{s}\right|$ is the corresponding Dynkin diagram $A_{n}, D_{n}, E_{6}, E_{7}$, or $E_{8}$.

Proof. We first note that since $R / m=k$ is algebraically closed, $\operatorname{End}(A) / \operatorname{rad} \operatorname{End}(A) \simeq k$ for each indecomposable $R$-module $A$. Since $R$ is Gorenstein of dimension 2, we know that $\mathrm{D} \operatorname{Tr} M \simeq M$ when $M$ is an indecomposable nonprojective reflexive module [3]. This shows that the arrows in the $A R$-quiver occur in pairs $\rightleftarrows \cdot$. By $[10]$ there is a preprojective partition for the category of finitely generated reflexive $R$-modules, and since we have finite representation type, every reflexive is preprojective. This implies that for every indecomposable $M$ there is a chain of irreducible maps from a projective module, that is, in this case from $R$. It follows that $\Gamma$ is connected.

A crucial point in our proof is that even though there is no almost split sequence with $R$ on the right, there is an exact sequence $0 \rightarrow R \stackrel{f}{\rightarrow} E \stackrel{g}{\rightarrow} R \rightarrow R / m \rightarrow 0[\mathbf{3}, \mathbf{4}]$, called the fundamental sequence in [4], such that $g: E \rightarrow R$ is minimal right almost split. It also follows directly or from [3] that $f: R \rightarrow E$ is minimal left almost split. This shows that $\mathrm{rk} E=2 \mathrm{rk} R=2$, where rk denotes rank, so that with respect to the additivity formula of ranks the fundamental sequence behaves like an almost split sequence. Hence by assigning to each vertex in the $A R$-quiver the rank of the corresponding module, we get an additive function for $|\Gamma|$, that is a function $\alpha$ on the vertices such that $2 \alpha(i)=\sum \alpha(j)$, where the sum is taken over all edges $i \cdot-\cdot j$. 
Then it is known (see [14]) that $|\Gamma|$ must be extended Dynkin $\tilde{A}_{n}, \tilde{D}_{n}, \tilde{E}_{6}, \tilde{E}_{7}, \tilde{E}_{8}$, or<smiles>CO</smiles>

or<smiles>CC(C)COO</smiles>

We want to exclude the last two cases. The corresponding additive functions are known to be an integral multiple of the following.

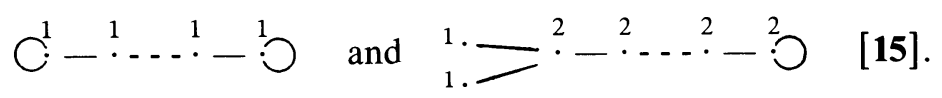

Since rk $R=1$, the listed ones would be the additive functions. The class group $C(R)$ of $R$ has as elements the isomorphism classes of indecomposable reflexive modules of rank 1. Since $R$ is assumed not to be regular local, there are at least two nonisomorphic indecomposable reflexive $R$-modules. Hence if one of the above cases occurs, $C(R)$ must be nontrivial. On the other hand $C(R)$ can be computed in a different way, using that $K_{0}(\bmod R) \simeq C(R) \amalg Z$, where $K_{0}(\bmod R)$ denotes the Grothendieck group of finitely generated $R$-modules modulo the relations generated by exact sequences (see [8]). In both cases we get one or two components of the form

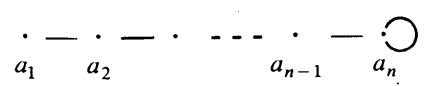

by removing $R$. Consider the free group on the vertices $a_{1}, \ldots, a_{n}$, modulo the relations generated by almost split sequences, that is $2 a_{1}-a_{2}, 2 a_{2}-a_{1}-$ $a_{3}, \ldots, 2 a_{n-1}-a_{n-2}-a_{n}, 2 a_{n}-a_{n-1}-a_{n}=a_{n}-a_{n-1}$. From this it follows that $C(R)=(0)$, a contradiction.

Removing $R$ means removing a vertex where the additive function given by ranks has value 1 . In each of the cases this gives for the extended Dynkin diagrams $\tilde{\Delta}$ the Dynkin diagram $\Delta$.

If for a category $\mathbf{L}$ having almost split sequences we drop the assumption $r_{A B}=s_{A B}$, we can consider the valued $A R$-quiver. Corresponding to an irreducible map from $A$ to $B$ we now write

$$
A \cdot \stackrel{\left(r_{A B}, s_{A B}\right)}{\longrightarrow} \cdot B
$$

If $R$ is as before, but without the assumption that $R / m$ is algebraically closed, we still have $\mathrm{DTr} A \simeq A$ for $A$ indecomposable not isomorphic to $R$. The valued arrows between nonisomorphic modules then occur in pairs

$$
A \cdot \underset{\left(s_{B A}, r_{B A}\right)}{\stackrel{\left(r_{A B}, s_{A B}\right)}{\rightleftarrows}} \cdot B
$$

where clearly $r_{A B}=s_{B A}, s_{A B}=r_{B A}$. Note that 
are the same $A R$-quivers. We then define $|\Gamma|$ to be the valued graph where each such pair is replaced by

$$
A \cdot \frac{\left(r_{A B}, s_{A B}\right)}{B} \cdot B
$$

and each

$$
\stackrel{(r, r)}{\Upsilon} \text { by } \overbrace{}^{r} \text {. }
$$

The arguments on the preprojective partition and the fundamental sequence are still valid. In this case the ranks give an additive function for the valued graph, defined in a natural way, and in addition to the previous possibilities we have the valued extended Dynkin diagrams or

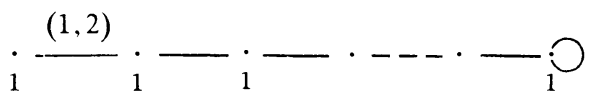

or

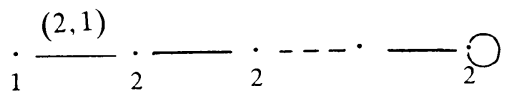

Here we have also listed the corresponding smallest additive function. The first of the last two cases can be excluded as before, but we do not know if the last one can occur.

Theorem 1 applies to rational double points over an algebraically closed field, since they are of finite representation type by [2], that is, have only a finite number of isomorphism classes of indecomposables in $\mathbf{L}$. In this case the resolution graph for the corresponding isolated singularity is a Dynkin diagram. Based on a formula which we shall next explain and which follows from Theorem 1 (or alternatively, part of the proof), a direct proof of an isomorphism between $\left|\Gamma_{s}\right|$ and the resolution graph is given by Ésnault-Knörrer in [13].

When $|\Gamma|$ is extended Dynkin, we have $r_{A B}=s_{A B}$. Since DTr is the identity on nonprojective indecomposable reflexives, we have an additivity relation at each nonprojective vertex. Using $|\Gamma|$ being extended Dynkin, it is easy to see that this forces an additivity relation also at the vertex of $R$. In the proof of Theorem 1 these statements followed from the "symmetry" for almost split sequences when $R / m$ is algebraically closed, and from the existence and properties of the fundamental sequence. If now for each indecomposable reflexive $A_{i}, B_{i} \rightarrow A_{i}$ denotes a minimal right almost split map, the desired formula used in [13] is

$$
\coprod_{i=1}^{n}\left(\operatorname{rk} A_{i}\right) B_{i} \simeq 2 \coprod_{i=1}^{n}\left(\operatorname{rk} A_{i}\right) A_{i} .
$$

Here $A_{1}, \ldots, A_{n}$ are all the indecomposable reflexives. Clearly a given $A_{i}$ occurs $2 \mathrm{rk} A_{i}$ times as a summand on the right. For each $A_{j}, A_{i}$ occurs on the left with multiplicity $t_{j}$ rk $A_{j}$, where $t_{j}$ is the multiplicity of $A_{i}$ in $B_{j}$. By our symmetry $t_{j}$ is 
then the multiplicity of $A_{j}$ in $C_{i}$, when $A_{i} \rightarrow C_{i}$ is minimal left almost split. Summing up, we then get $2 \mathrm{rk} A_{i}$, by additivity.

If we drop the asumption that $R / m=k$ is algebraically closed, the formula holds if and only if the (smallest) additive function for $|\Gamma|$ is also an additive function for the valued graph obtained by replacing $A \cdot \frac{(r, s)}{B} B$ by $A \cdot \frac{(s, r)}{B} \cdot B$. By considering the different cases, listed in [15], we must then have $r=s$ for all edges, so that we are back in the situation when $|\Gamma|$ is extended Dynkin. Hence the formula does not hold in general when $R / m$ is not algebraically closed. It holds, however, for the noncommutative two-dimensional rings treated in §3. In other dimensions we do not have fundamental sequences.

We now make some remarks on the construction of almost split sequences for rational double points. It is shown in [4] (in a generalized version) that if

$$
0 \rightarrow R \rightarrow E \rightarrow R \rightarrow R / m \rightarrow 0
$$

is the fundamental sequence, then for an indecomposable nonprojective reflexive $R$-module $A, 0 \rightarrow A \rightarrow E \cdot A \rightarrow A \rightarrow 0$ is almost split if and only if char $k+\operatorname{rk} A$. If char $k \mid \mathrm{rk} A$, the sequence splits. Here $E \cdot A=\left(E \otimes_{R} A\right)^{* *}$, where $X^{*}=$ $\operatorname{Hom}_{R}(X, R)$. Since by considering the smallest additive functions for extended Dynkin diagrams we know that $\mathrm{rk} A \leqslant 6$, all almost split sequences can be computed this way when char $k>5$. We note, however, that except for the case $D_{n}$ for char $k=2$, this principle can still be used to compute the almost split sequences. For assume that for some indecomposable $B$, char $k \mid$ rk $B$. Except for the case mentioned, for all neighbors $A_{i}$ of $B$ in $|\Gamma|$, char $k+\operatorname{rk} A_{i}$. Hence we have almost split sequences $0 \rightarrow A_{i} \rightarrow E \cdot A_{i} \rightarrow A_{i} \rightarrow 0 . B \mid E \cdot A_{i}$, and the induced map $A_{i} \rightarrow B$ is irreducible, and we get a minimal right almost split map $\amalg A_{i} \rightarrow B$.

We end this section with observing that knowing that $|\Gamma|$ is extended Dynkin gives information on the relationship between rank and minimal number of generators.

PROPOSITION 2. Let $R$ be a rational double point over an algebraically closed field, and let $A$ be an indecomposable reflexive module not isomorphic to $R$. Then the minimal number of generators of $A$ is $2 \mathrm{rk} A$.

Proof. $\Omega$ induces an equivalence on the stable category, and as in the finitedimensional case [7], a map between indecomposable nonprojectives is irreducible if and only if it is irreducible in the stable category. Hence $\Omega$ induces an automorphism on $\Gamma_{s}$. By considering the additive functions, we see that $\Omega$ must preserve rank. Our claim then follows by considering $0 \rightarrow \Omega M \rightarrow P \rightarrow M \rightarrow 0$.

2. Generalizations. In this section we first give a generalization of Theorem 1 to the two-dimensional noncommutative case, and for this we recall the definition of order and lattice [3]. \$3 will shed some more light on this. We also consider the two-dimensional case when $D \operatorname{Tr}$ is not necessarily the identity, and also higher dimensions. 
Let $T$ be a complete regular local two-dimensional ring, and $\Lambda$ an indecomposable $T$-order, that is, $\Lambda$ is a finitely generated free $T$-module such that $\operatorname{Hom}_{T}(\Lambda, T)_{k}$ is $\Lambda_{h}$-projective for all nonmaximal prime ideals $\not h$ in $T$. A $\Lambda$-module $L$ is said to be a lattice if $L$ is a finitely generated free $T$-module such that $L_{p}$ is $\Lambda_{p}$-projective for all nonmaximal prime ideals $\not 2$ [3]. Then we know that the category of lattices $\mathbf{L}$ has almost split sequences, and in this case $\tau=D \operatorname{Tr}$ is given by $\tau(X)=$ $\operatorname{Hom}_{T}\left(\operatorname{Hom}_{\Lambda}(X, \Lambda), T\right)$ [3]. Considering $\tau$ as a functor $\tau: \bmod \Lambda \rightarrow \bmod \Lambda$, we see that $\tau$ is right exact, and hence $\tau \simeq \operatorname{Hom}_{T}(\Lambda, T) \otimes_{\Lambda}-$. It is important that if $P$ is indecomposable projective, then we have an exact sequence

$$
0 \rightarrow \tau P \stackrel{f}{\rightarrow} E \stackrel{g}{\rightarrow} P \rightarrow P / \operatorname{rad} P \rightarrow 0,
$$

such that $g$ is minimal right almost split, and $f$ is minimal left almost split [3]. Using ranks (over $T$, or when possible, over $\Lambda$ ), this provides us with additivity relations as before.

As in the commutative case, the situation is especially nice when $\tau$ is the identity on indecomposable lattices. This is for example the case when $\Lambda$ is a Gorenstein $R$-order, that is, we have a bimodule isomorphism $\Lambda \simeq \operatorname{Hom}_{T}(\Lambda, T)$. In $\S 3$ we will see interesting examples where $\Lambda$ is not Gorenstein, but still $\tau=\mathrm{id}$ for indecomposable lattices.

If there is only one indecomposable projective lattice, it follows as before by using the preprojective partition that the $A R$-quiver $\Gamma$ is connected. With the same arguments as before, where for simplicity we state the result for the case of nonvalued quivers, we get the following.

THEOREM 3. Let $T$ be a complete regular local two-dimensional ring with maximal ideal $m$, and $\Lambda$ a $T$-order of finite lattice type. Assume that $\tau=\mathrm{id}$ on indecomposable lattices, and that $T / m$ is an algebraically closed field $k$. Then the following hold for the AR-quiver $\Gamma$ and the stable AR-quiver $\Gamma_{s}$.

(a) A connected component of $|\Gamma|$ is extended Dynkin or

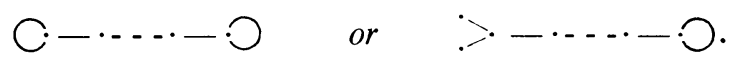

(b) A connected component of $\left|\Gamma_{s}\right|$ is Dynkin or

In $\$ 3$ we shall see that contrary to the commutative two-dimensional case, the diagrams with loops actually do occur.

As before, there are restrictions on the valued graphs when we drop the assumption on $T / m$.

Even if $\tau$ is not the identity, considering ranks still gives additivity relations in the two-dimensional case. If there are no loops in the $A R$-quiver $\Gamma$ and we have finite lattice type, combinatorics shows that $\Gamma$ is of the form $Z \Delta / G$, where $Z \Delta$ is a translation quiver for an oriented tree $\Delta$ whose underlying graph is extended Dynkin or $A_{\infty}$, and $G$ is a finite group (see $[18,14]$ ). An explicit computation for the commutative rings of the form $\Lambda=k[[X, Y]]^{G}$, where $G \subset \mathrm{GL}(2, k)$ is a finite 
group and $k$ is an algebraically closed field with char $k=0$, is given in [9]. Other examples when $\tau$ is not the identity are given in $\S 3$.

For higher dimensions we do not have so satisfactory results since we do not have "almost split sequences" for the projectives. Then, if DTr is the identity, the combinatorics only applies to $\left|\Gamma_{s}\right|$. For this recall that for a given graph a function $\alpha$ on the vertices is subadditive if for every vertex $i, 2 \alpha(i) \geqslant \sum \alpha(j)$, where the sum runs over all edges $i \cdot-\cdot j$. Let now $R$ be a complete local integrally closed isolated hypersurface singularity with $R / m$ algebraically closed. Then $\mathrm{D} \operatorname{Tr} M \simeq \Omega^{2-2 n} M$ by [3] and $\Omega^{2} M \simeq M$ by [12], where $n=\operatorname{dim} R$. Hence DTr is the identity for $M \neq R$ in even dimensions, and also in some cases in odd dimensions. In these cases the rank function gives a subadditive function on $\left|\Gamma_{s}\right|$ which is not additive. Combinatorics then gives that if $R$ is of finite lattice type, that is, has only a finite number of isomorphism classes of indecomposable Cohen-Macaulay modules [5], the connected components of $\left|\Gamma_{s}\right|$ are Dynkin or

Now we point out that if char $k \neq 2$ it is known from [16] that $\left|\Gamma_{s}\right|$ is actually Dynkin in the even-dimensional case, and the additional diagram occurs in any size in any odd dimension. In the odd-dimensional case $\mathrm{D} \operatorname{Tr} M \simeq \Omega M$ for $M \neq R$, and since $\Omega^{2} M \simeq M, \Gamma_{s}$ has a periodic vertex. Hence when there is no loop in $\Gamma_{s}$, $\Gamma_{s} \simeq Z \Delta / G$, where $Z \Delta$ is built from a Dynkin diagram $\Delta$ [14].

We point out that there is information along the same lines for infinite representation type, since there are still combinatorial results for additive and subadditive functions [15].

3. Computations for two-dimensional orders. Let $T$ be the power series ring $T=k[[u, v]]$, where $k$ is an algebraically closed field with char $k=0$. Let $A$ be a maximal $T$-order with center $T$ in a division ring $D$, and assume that $A$ is not commutative. In [1] Artin has given a description of when $A$ has only a finite number of isomorphism classes of indecomposable $A$-modules which are free $T$-modules. $A$ is then a $T$-order in the sense of [3], and the $A$-modules which are free $T$-modules are the lattices. On the basis of Artin's classification, we compute the $A R$-quivers when $A$ is of finite representation type. In some cases the equivalence

$$
\tau=\operatorname{Hom}_{T}\left(\operatorname{Hom}_{\Lambda}(, \Lambda), T\right): \mathbf{L}(A) \rightarrow \mathbf{L}(A)
$$

induces the identity on indecomposable objects, in other cases not. From the point of view of this paper the most interesting aspect of these computations is that both cases with loops which we could exclude in the commutative case actually do occur. At the same time we see that there is a close connection between the $A R$-quivers and the $A$-diagrams studied by Artin, thus giving an interpretation of Artin's diagrams in most cases.

We investigate the classes classified by Artin case by case (when there are at least two indecomposables). We use his description of indecomposables of $A$-rank 1 , and the total distribution of ranks for all indecomposables. Note that the $A R$-quiver is 
connected since there is only one indecomposable projective lattice, by the argument using preprojective partitions as before.

Type $\left(\mathrm{II}_{k}\right) . A$ is generated over $T$ by $x, y$, satisfying

$$
x^{2}=u, \quad y^{2}=u^{k} v, \quad x y+y x=2 v .
$$

Here all indecomposable lattices have rank 1, and the nonprojective ones are $L_{i}=\left(u^{i}, y\right), 1 \leqslant i \leqslant k$.

We first observe that $\tau A \simeq A$. To see this, consider the $T$-basis $\{1, x, y, x y\}$ for $A$ as ieft $T$-module, and let $f_{x y}: A \rightarrow T$ be the $T$-map given by

$$
f_{x y}\left(r_{1}+r_{2} x+r_{3} y+r_{4} x y\right)=r_{4} .
$$

Then $\tau(A)=\operatorname{Hom}_{T}(A, T)=A f_{x y} \simeq A$.

Let $f: A \rightarrow A$ be given by $f(a)=a x$, let $g: L_{1}=(u, y) \rightarrow A$ be the inclusion, and consider the induced map $(f, g): A \amalg L_{1} \rightarrow A$. It is not hard to get a good enough description of an arbitrary map $h: L_{i} \rightarrow A$ to see that it can be lifted to $A \amalg L_{1}$. Since $\operatorname{Im}(f, g)=(x, y)$, any nonisomorphism $h: A \rightarrow A$ can also be lifted. This shows that $(f, g): A \amalg L_{1} \rightarrow A$ is right almost split, and a rank argument shows that it is minimal right almost split, so that we have the "almost split sequence" $0 \rightarrow A \rightarrow A \amalg L_{1} \rightarrow A$. Applying $\tau$, we see that $\tau L_{1} \simeq L_{1}$. Hence we have an almost split sequence $0 \rightarrow L_{1} \rightarrow A \amalg X \rightarrow L_{1} \rightarrow 0$. Applying $\tau$ again shows that $\tau X \simeq X$. Continuing, and using that the $A R$-quiver $\Gamma$ is connected, we get that $\tau$ is the identity on objects, and $|\Gamma|$ is

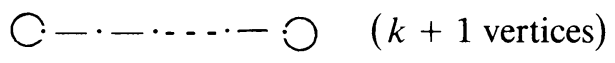

and $\left|\Gamma_{s}\right|$ is

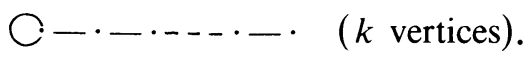

Type $\left(\mathrm{III}_{k}\right) . A$ is generated over $T$ by $x, y$, where

$$
x^{2}=v, \quad y^{2}=u v, \quad x y+y x=2 u^{k+1} .
$$

Here there is only one nonprojective lattice $L$ of rank 1 , and the $k$ others are of rank 2.

$\{1, x, y, x y\}$ is $T$-basis for $A$ as a left $T$-module, and it is easy to see that $\tau(A)=\operatorname{Hom}_{T}(A, T)=A f_{x y} \simeq A$, and $\operatorname{Hom}_{T}(A, T) \simeq f_{x y} A \simeq A$. Denoting also by $\tau$ the automorphism given by $\tau(a)=a^{\prime}$, when $a f_{x y}=f_{x y} a^{\prime}$, we have $\tau(X)=X^{\tau}$. Here $X^{\tau}$ denotes the $\Lambda$-module with the same underlying group as $X$, and where $\lambda \cdot x=\tau(\lambda) x$. Since $\tau$ clearly preserves rank, we have $\tau L \simeq L$. Consider the "almost split sequence" $0 \rightarrow A \rightarrow K \rightarrow A$. Applying $\tau$, we conclude $\tau K \simeq K$. Assume that $K \simeq K_{1} \amalg K_{2}$, where $K_{1}$ and $K_{2}$ both have rank 1 . The only possibilities for $K_{1}$ and $K_{2}$ are $A$ and $L$, and in any case we would get a connected component of the $A R$-quiver with all indecomposables having rank 1 . It follows that $K$ is indecomposable. Consider the almost split sequence $0 \rightarrow K \rightarrow A \amalg X \rightarrow K \rightarrow 0$. $X$ has rank 3, so $X$ has one indecomposable summand of rank 1 and one of rank 2 . Since $A / m=k$ is algebraically closed, where $m$ is the maximal ideal of $A, A$ is not 
a summand of $X$, and hence we have almost split sequences

$$
0 \rightarrow K \rightarrow A \amalg L \amalg K_{1} \rightarrow K \rightarrow 0
$$

and $0 \rightarrow L \rightarrow K \rightarrow L \rightarrow 0$. This shows that $\tau\left(K_{1}\right) \simeq K_{1}$. If $K_{1} \simeq K$, we are done. If $K_{1} \neq K$, we have the almost split sequence $0 \rightarrow K_{1} \rightarrow K \amalg K_{2} \rightarrow K_{1} \rightarrow 0 . K_{2}$ can have no summand of rank 1 , so $K_{2}$ is indecomposable of rank 2 , and $\tau K_{2} \simeq K_{2}$ follows. Continuing this way, we see that $\tau=\mathrm{id}$ on all indecomposable lattices, and $|\Gamma|$ is

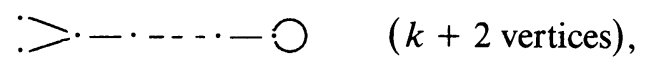

and $\left|\Gamma_{s}\right|$ is

$$
\cdot-\cdot-\cdot-\bigcirc \quad(k+1 \text { vertices }) .
$$

Type (IV). $A$ is generated over $T$ by $x, y$, where

$$
x^{3}=v, \quad y^{3}=u^{2}-v, \quad x y=\rho_{3} y x,
$$

$\rho_{3}$ a primitive third root of 1 . The indecomposable nonprojective lattices of rank 1 are $L_{1}=(u, x+y), L_{2}=\left(u, x+\rho_{3} y\right)$ and $L_{3}=\left(u, x+\rho_{3}^{2} y\right)$. There is only one additional indecomposable lattice $L$, of rank 2 .

$\left\{1, x, y, x^{2}, y^{2}, x y, x y^{2}, x^{2} y, x^{2} y^{2}\right\}$ is a $T$-basis for $A$ as a left $T$-module. An easy computation gives $\operatorname{Hom}_{T}(A, T)=A f_{x^{2} y^{2}}=f_{x^{2} y^{2}} A$, and that the automorphism $\tau: A \rightarrow A$ is determined by $\tau(x)=\rho_{3}^{2} x, \tau(y)=\rho_{3} y$. We further can compute $\tau\left(L_{1}\right) \simeq L_{2}, \tau\left(L_{2}\right) \simeq L_{3}, \tau\left(L_{3}\right) \simeq L_{1}$.

Using this, it is not hard to compute the $A R$-quiver. We have $\tau A \simeq A$, and an "almost split sequence" $0 \rightarrow A \rightarrow E \rightarrow A$, and hence $\tau E \simeq E$. Clearly $E \neq A \amalg A$, and also no $L_{i}$ can be a summand of $E$. Hence $E$ must be the indecomposable of rank 2. Further we have the almost split sequence $0 \rightarrow E \rightarrow A \amalg F \rightarrow E \rightarrow 0$, where $A$ is not a summand of $F$. Since $F$ has rank 3, one of the $L_{i}$ must then be a summand of $F$. Applying $\tau$ we get that we must have

$$
0 \rightarrow E \rightarrow A \amalg L_{1} \amalg L_{2} \amalg L_{3} \rightarrow E \rightarrow 0,
$$

and hence we have almost split sequences $0 \rightarrow \tau\left(L_{i}\right) \rightarrow E \rightarrow L_{i} \rightarrow 0$. The $A R$-quiver is then

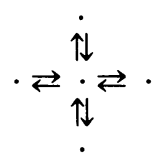

with $\tau$ permuting three end vertices cyclically. $|\Gamma|$ is then

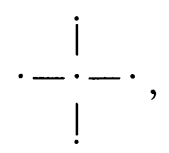

which is $\tilde{D}_{4}$. 
Type (V). $A$ is generated over $T$ by $x, y$, where

$$
x^{2}=v, \quad y^{2}=v^{2}-u^{3}, \quad x y=-y x .
$$

There are two nonprojective indecomposables of rank $1, L_{1}=(u, y+v)$ and $L_{2}=(u, y-v)$. In addition there are three indecomposables of rank 2 and one of rank 3.

Here $\{1, x, y, x y\}$ is a $T$-basis for $A$ as left $T$-module, and we have

$$
\operatorname{Hom}_{T}(A, T) \simeq A f_{x y}
$$

as left $A$-module, so that $\tau(A) \simeq A$. Also $\operatorname{Hom}_{T}(A, T) \simeq f_{x y} A$, and an easy computation shows that $\tau$ is induced by $\tau(x)=-x, \tau(y)=-y$. One can then further compute that $\tau\left(L_{1}\right) \simeq L_{2}, \tau\left(L_{2}\right) \simeq L_{1}$.

Consider the "almost split sequence" $0 \rightarrow A \rightarrow E \rightarrow A$. If $E$ decomposes, we get a component with indecomposables of rank 1 . Hence $E$ is indecomposable and $\tau E \simeq E$. Consider the almost split sequence $0 \rightarrow E \rightarrow A \amalg F \rightarrow E \rightarrow 0 . A$ is not a summand of $F$, and since $\tau F \simeq F$, the indecomposable summands of $F$ cannot all be of rank 1. Also there cannot be exactly one of rank 1 . It follows that $F$ is indecomposable of rank 3. Consider now the almost split sequence

$$
0 \rightarrow F \rightarrow E \amalg G \rightarrow F \rightarrow 0 .
$$

A rank argument shows that $G$ has no summand of rank 1, and consequently none of rank 3. Hence the indecomposable summands of $G$ must have rank 2, and since there can be no repeats, we have $0 \rightarrow F \rightarrow E \amalg E^{\prime} \amalg E^{\prime \prime} \rightarrow F \rightarrow 0$, where $E, E^{\prime}, E^{\prime \prime}$ are the three indecomposables of rank 2. It now follows that the $A R$-quiver is

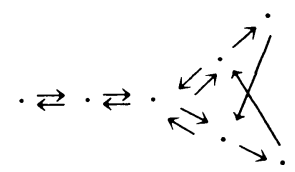

From the point of view of this paper, the computations for $\left(\mathrm{II}_{k}\right)$ and $\left(\mathrm{III}_{k}\right)$ are most interesting, since $\tau=\mathrm{id}$, and

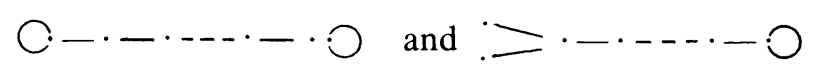

occur for $|\Gamma|$, for any size of these diagrams. (The rings are, however, not Gorenstein.) These diagrams also coincide with the $A$-diagrams of Artin in these cases, thus providing an interpretation of these $A$-diagrams. This is still the case for (IV). For (V) Artin's $A$-diagram is $\tilde{E}_{6}$, which is closely connected with the $A R$-quiver $\Gamma$, since $\Gamma$ is of the form $Z \Delta / G$, where $\Delta$ is an oriented tree of type $\tilde{E}_{6}$.

\section{REFERENCES}

1. M. Artin, Maximal orders of global dimension and Krull dimension two, Invent. Math. 84 (1986), $195-222$.

2. M. Artin and M. L. Verdier, Reflexive modules over rational double points, Math. Ann. 270 (1985), $79-82$

3. M. Auslander, Functors and morphisms determined by objects, Proc. Conf. on Representation Theory (Philadelphia, 1976), Dekker, New York, 1978, pp. 1-244. 
4. , Rational singularities and almost split sequences, Trans. Amer. Math. Soc. 293 (1986), 511-531.

5. _ Isolated singularities and existence of almost split sequences, Proc. ICRA IV, Lecture Notes in Math., vol. 1178, Springer, 1986, pp. 194-241.

6. M. Auslander and I. Reiten, Representation theory of artin algebras III: Almost split sequences, Comm. Algebra 3 (1975), 239-294.

7. Stable equivalence of artin algebras, Proc. Conf. on Orders, Group Rings and Related Topics, Lecture Notes in Math., vol. 353, Springer, 1972, pp. 8-71.

8. __ Grothendieck groups of algebras and orders, J. Pure Appl. Algebra 39 (1986), 1-51.

9. __ McKay quivers and extended Dynkin diagrams, Trans. Amer. Math. Soc. 293 (1986), 293-302.

10. M. Auslander and S. O. Smalø, Lattices over orders: Finitely presented functors and preprojective partitions, Trans. Amer. Math. Soc. 273 (1982), 433-446.

11. R. Bautista, Irreducible maps and the radical of a category, An. Inst. Mat. Univ. Nac. Autonoma México 22 (1982), 83-135.

12. D. Eisenbud, Homological algebra on a complete intersection, with an application to group representations, Trans. Amer. Math. Soc. 260 (1980), 35-64.

13. H. Esnault and H. Knörrer, Reflexive modules over rational double points, Math. Ann. 272 (1985), 545-548.

14. D. Happel, U. Preisel and C. M. Ringel, Vinberg's characterization of Dynkin diagrams using subadditive functions with applications to DTr-periodic modules, Proc. ICRA II, Ottawa 1979, Lecture Notes in Math., vol. 832, Springer, 1980, pp. 280-294.

15. __ Binary polyhedral groups and Euclidean diagrams, Manuscripta Math. 31 (1980), 317-329.

16. H. Knörrer, Cohen-Macaulay modules on hypersurface singularities I (preprint)

17. J. McKay, Graphs, singularities and finite groups, Proc. Sympos. Pure Math., vol. 37, Amer. Math. Soc., Providence, R. I., 1980, pp. 183-186.

18. C. Riedtmann, Algebren, Darstellungsköcher und Zurück, Comment. Math. Helv. 55 (1980), 199-224.

Department of Mathematics, Brandeis University, Waltham, Massachusetts 02254

Institute for Mathematics and Statistics, University of Trondheim, AVH, 7055 Dragvoll, NORWAY (Current address of Idun Reiten)

Current address (Maurice Auslander): Department of Mathematics, Virginia Polytechnic Institute and State University, Blacksburg, Virginia 24061-4097 